\title{
Effect of Regular Physical Exercise on Gut Microbiota and Depressive Behaviors in Rats
}

\author{
Liangju Sheng, Yuxuan Wang, Anqi Jiang, Yan Zhou, and Hong Zhou \\ School of Medicine, Jiangsu University, Zhengjiang 212013, Jiangsu, China \\ Correspondence should be addressed to Hong Zhou; hongzhou0123@163.com
}

Received 8 August 2021; Accepted 18 September 2021; Published 5 October 2021

Academic Editor: Fatma M. El-Demerdash

Copyright (c) 2021 Liangju Sheng et al. This is an open access article distributed under the Creative Commons Attribution License, which permits unrestricted use, distribution, and reproduction in any medium, provided the original work is properly cited.

Objective. The gut microbiota, as the critical mediator of the gut-brain axis, can produce and transport neuroactive substances, thus playing a significant role in the pathogenesis of depression. Although regular physical exercise is an important nondrug antidepressant, its specific effector mechanism is still unclear. Methods. Rats were randomly divided into four different groups ( $n=10$ for each group) as follows: normal group (G1), depression group (G2), fluoxetine treatment group (G3), and regular exercise treatment group (G4). All rats underwent forced swimming tests, tail suspension tests, open field tests, and elevated plusmaze tests to detect behavioristics. Then, corticosterone levels were detected by ELISA. Additionally, taxonomic analysis of the gut microbiota in all rats was performed after they were exercised regularly for 60 days. Results. Compared with the G1 group, the rats in the G2 group showed significant depression-like behaviors, with increased serum corticosterone levels. The proportions of Bacteroides, Actinomycetes, Proteobacteria, Saccharomyces, and Cyanobacteria in rats of the G2 group were lower than those in the G1 group, while the proportions of Firmicutes, Tenicotte, Deferrobacteria, and Fusobacteria were increased. Furthermore, after regular exercise treatment, the gut microbiota of rats was effectively improved, almost returning to the level of the G1 group, and depressive behavior and corticosterone levels were also restored, which was almost the same as the effect of fluoxetine treatment. Conclusion. Regular physical exercise could alleviate depressive-like behaviors by modulating the species and function of the gut microbiota.

\section{Introduction}

Depression is a serious mental disorder that can be accompanied by a strong sense of guilt, helplessness, changes in appetite and sleep, and damage to organ functions. Severe depression can even lead to self-mutilation and suicide [1]. Recent studies have shown that there is a bidirectional pathway between the gut and the central nervous system, also known as the gut-brain axis, which is closely related to the complex crosstalk of the autonomic nervous system, neuroendocrine system, and immune system [2, 3].

On the one hand, increasing evidence suggests that gut microbiota is a critical environmental factor. It can produce and transport neuroactive substances, which are considered the key mediators of the gut-brain axis and play an important role in the mechanism of depression [4]. The past studies showed that transplanting the MDD patients' gut microbiota into germ-free mice led to depressive behavior [5], and transplanting the gut microbiota from depressive patients into rats subjected to antibiotic treatment also replicated depressive behavior [6]. These results all proved that changes in the composition of the gut microbiota may be an important factor leading to depression. Further research studies showed that gut microbiota can generate a pathological feed-forward loop that contributes to depressive-like behaviors via the central endocannabinoid (eCB) system [7]. The mediation of the gut microbiota through host's metabolism can also induces depressive-like behaviors [5].

On the other hand, regular physical exercise is an important nondrug antidepressant method, but its specific mechanism is still unclear $[8,9]$. Therefore, this article intends to use depression model rats as the object to dynamically observe whether regular physical exercise can 
improve the prevalence and composition of the gut microbiota of rats, thereby exerting an antidepressant effect, with the aim of providing relevant theoretical references for the treatment of depressive diseases.

\section{Materials and Methods}

2.1. Animal Studies. Forty SD rats (female, $180 \mathrm{~g}$ ) provided by the experimental animal center of Jiangsu University (Zhenjiang, China) were used in this study. The animals were housed under standard conditions $\left(22 \pm 1^{\circ} \mathrm{C}\right.$ room temperature, $55 \% \pm 1 \%$ humidity, and $12 \mathrm{~h}$ light/dark cycles) in regular cages and allowed free access to food and water. All animal experiments were performed according to the Guidelines for the Care and Use of Laboratory Animals by the National Institutes of Health. Approvals for the study were acquired from the Ethics Committee of the Medical College of Jiangsu University (Zhenjiang, China).

2.2. Study Design. Rats were randomized into four groups (10 rats in each group): a normal group (G1), a depression group (G2), a fluoxetine treatment group (G3), and a regular exercise treatment group (G4), with 10 rats in each group. In G2, G3, and G4, chronic unpredictable mild stress (CUMS) was employed for 4 weeks. In brief, the stimuli included tail pinching for 1 minute with a clip ( 8 am to $10 \mathrm{am}$, just until the rats whined, without skin damage), swimming in $6^{\circ} \mathrm{C}$ cold water for 5 minutes ( 8 am to 11 am, using a $25 \mathrm{~cm}$ high plastic drum that was $15 \mathrm{~cm}$ in diameter, with the water depth determined by the rats' toes reaching the bottom of the container), bound for 2 hours, housed in an empty cat cage for 15 hours ( 5 pm to 8 am of next day) and exposed to light overnight, and lack of food or water for 24 hours ( 8 am to 8 am of next day). These stressors were applied in random order in 1 day. Each stressor was repeated two or three times during the four-week stress period. G3 was given fluoxetine $3 \mathrm{mg} / \mathrm{kg}$ daily for 3 weeks by gavage, and in G4, Sierakowiak et al.' protocol was used [10]. The depressed model rats were placed in a $35 \mathrm{~cm}$ motorized wheel and exercised for 1 hour at 9 am and 3 pm each day.

\subsection{Rat Behavioral Tests}

2.3.1. Forced Swim Test (FST). The test rat was individually placed in a plexiglass cylinder with a height of $25 \mathrm{~cm}$ and a diameter of $15 \mathrm{~cm}$ with a water depth of $10 \mathrm{~cm}$ and a water temperature of $25 \pm 1^{\circ} \mathrm{C}$. After $15 \mathrm{~min}$ of pretesting for 3 days, the rats' swimming time, struggling time, and static floating time in the water within 5 minutes were recorded.

2.3.2. Tail Suspension Test (TST). The test rat was suspended $40 \mathrm{~cm}$ above the table with tape, and the tape was placed approximately $1 \mathrm{~cm}$ from the tip of the tail. In the 6 -minute test, the resting time of the rat, which is the total time that the rat exhibited no limb or body movements, was recorded.
2.3.3. Open Field Test (OFT). The apparatus for the open field test was a box $(60 \mathrm{~cm} \times 60 \mathrm{~cm} \times 30 \mathrm{~cm})$. The rats were exposed to dark or dim conditions and reared alone in a box. The movement of the rats in the container was represented by movement distance and was monitored by an S-Mart computerized video tracking system. The number of times crossing the line and the distance walked in the container were recorded.

2.3.4. Elevated Plus-Maze Test (EPM). The test device consisted of two oppositely placed open arms $(50 \mathrm{~cm} \times 10 \mathrm{~cm})$ and two oppositely placed closed arms $(50 \mathrm{~cm} \times 10 \mathrm{~cm} \times 20 \mathrm{~cm})$ connected by a central platform $(10 \mathrm{~cm} \times 10 \mathrm{~cm})$. The maze was $50 \mathrm{~cm}$ above the ground and was made of black plexiglass. The rats were placed in the center of the maze facing the closed arm, and the frequency of the rat entering the open arm and the percentage of time spent in the open arm were recorded.

2.4. Assessment of Corticosterone Level. The collected blood samples were centrifuged at $3000 \mathrm{RPM}$ for $15 \mathrm{~min}$ at $4^{\circ} \mathrm{C}$ to collect serum, and the serum was stored in a refrigerator at $-80^{\circ} \mathrm{C}$ for later use. An ELISA kit was used to measure the level of corticosterone using a double-antibody sandwich enzyme-linked immunosorbent assay (ELISA) to detect the serum corticosterone content of rats in each group according to the kit's operating instructions. The absorbance was measured at $450 \mathrm{~nm}$, and the intensity of the color reaction was directly proportional to the concentration of the target protein in the sample.

2.5. DNA Extraction and Sequencing of the 16S rRNA Gene. To classify and analyze the microbial distribution, stool samples from 5 rats in each group were collected after 60 days of exercise and stored in a refrigerator at $-70^{\circ} \mathrm{C}$. The FastDNA Spin Kit for Soil was used to extract bacterial genomic DNA from 100 to $200 \mathrm{mg}$ of stool according to the manufacturer's instructions. The NanoDrop method was used to detect the purity of the extracted genomic DNA, and the Qubit dsDNA BR kit was used to detect the concentration of genomic DNA. Gene sequencing of 16S rRNA is based on the Illumina $16 \mathrm{~S}$ metagenomic sequencing library preparation guide. The forward primer of the $\mathrm{V} 4$ region (CCA GCM GCC GCG GTA ATW C) and the reverse primer of the V5 region (CC GTC AAT TYY TTT RAG TTT) were used to amplify the V4-V5 region of the bacterial $16 \mathrm{~S}$ rRNA gene. The amplified sequencing library was purified using AMPure XP magnetic beads, and the quality of the library was checked with a 2100 bioanalyzer. The sequencing library was mixed at equimolar concentrations to generate a $4 \mathrm{nM}$ library pool and was then sequenced on a MiSeq system with 250 bp paired-end reads using the MiSeq v2 kit.

2.6. Analysis of Sequencing Data. QIIME (v1.9.1) was used for data quality control and original sequence analysis. First, the original sequences were preprocessed and clustered into 
OTUs with $97 \%$ consistency by UCLUST software. Based on the Greengenes database (V13_8), classification was performed with 97\% consistent clustering, generating an OTU table and classification summary to provide a representation of the proportion of taxa in each sample. To analyze the diversity of bacteria, the representative sequences of OTUs were compared with PyNAST, and a phylogenetic tree was constructed with FastTree. A simplified OTU table was used to calculate the $\alpha$-diversity based on the observed species parameter and the Chaol, Shannon, and Simpson parameters. The unweighted UniFrac algorithm was used to calculate the $\beta$-diversity and was represented in the principal coordinate analysis (PCoA) graph.

2.7. Data Analysis. Quantitative data with a normal distribution are expressed as the means \pm standard deviation (SD). The Mann-Whitney nonparametric test was used to analyze all data. All statistical tests were 2 -sided, and $P<0.05$ was regarded as significant. Statistical analyses of data were performed using SPSS and GraphPad Prism 6.0.

\section{Results}

3.1. Regular Exercise Significantly Influenced the Behaviors of Rats. Compared with the rats in G2, the rats in G4 showed more frequent struggling behaviors (Figure 1(a)). The swimming behaviors of rats in G4 and G3 were significantly improved (Figure 1(b)). In the FST, the resting time of rats in the G2 group increased, while the resting times of rats in G3 and G4 were significantly reduced (Figure 1(c)). Physical exercise in rats also reduced the resting time in the TST (Figure 1(d)). In the open field experiment, the rats in G1 were active in the entire area, including the peripheral and central areas, while the movement of rats in G2 was significantly reduced in the peripheral and central areas (Figure 2(a)). Compared with the rats in G2, the total movement distance of the rats in G3 increased significantly, and the total movement distance of the rats in G4 also increased (Figure 2(b)). In addition, the movement distances of the rats in G4 and G3 in the surrounding area were greater than that of the rats in G2 (Figure 2(c)). The movement distance of the rats in G4 in the central area was significantly increased compared with that of the rats in G2, and the movement distance of the rats in G3 in the central area also increased similarly (Figure 2(d)). In the elevated plus-maze test, compared with the rats in G2, the rats in G3 and G4 spent less time in the closed arms and more time in the open arms (Figures 2(e) and 2(f)).

3.2. Regular Exercise Effectively Reduced the Level of Serum Corticosterone. Compared with G1, the serum corticosterone level of G2 was significantly higher. Moreover, the serum corticosterone levels in G4 was significantly lower than that in G2. In addition, the level of serum corticosterone in G3 was also decreased significantly. These results indicate that regular exercise can effectively reduce the production of serum corticosterone in rats (Figure 3).
3.3. Regular Exercise Had an Impact on the Diversity of Gut Microbiota. The stool samples were sequenced and analyzed by MiSeq and QIIME. The 16S rRNA gene sequencing of each sample produced more than 120,000 reads, and QIIME processed more than 7700 OTUs. To evaluate the ecological characteristics of these four groups of bacteria, we used different parameters to evaluate the diversity. In the $\alpha$-diversity, the observed species parameter and the Chaol parameter, which are the estimated values of species richness, only slightly increased in rats of G2, and the Shannon and Simpson parameters, which are the estimated values of species diversity, also showed similar trends (Table 1). Regarding $\beta$-diversity, the microbial flora compositions of rats in G1 and G2 were significantly different, while the microbial flora compositions of the rats in G3 and G4 were similar to that of the rats in G1 (Figure 4).

\subsection{Regular Exercise Had an Impact on the Different Microbial} Compositions in the Gut. The classification analysis at the microbial composition phylum level indicated that the composition of the gut microbiota was reshaped due to stress (Figure 5(a)). In the gut of G2 rats, the proportions of Bacteroides, Actinomycetes, Proteobacteria, TM2, and Cyanobacteria decreased, while the proportions of Firmicutes, Bacteroides, Deferrobacteria, and Fusobacteria increased (Figure 5(b)). Regular exercise helped restore the microbial composition of Actinomycetes and Cyanobacteria that were altered by stress by regulating the gut microbiota. At the genus level, $10^{5}$ genera in the gut of rats in G2 showed increasing or decreasing proportions, but in the rats in G3 and G4, 85\% of the gut microbiota was restored. Namely, S24-7_unclassified bacteria, Lactobacillus, traveling Bacillus, Bifidobacterium, and Desulfovibrio were reduced in relative abundance, while Bacteroides, Prevotella, Parabacteria, unclassified Bacillus, Rumenococcus, Doria, oscillating Spirulina, unclassified Rumenococcus, Corynebacterium, and Clostridium were increased in the gut of rats in G2. However, the rats in the G4 group showed recovery of the gut microbiota at the genus level, that is, the proportions of S247_unclassified bacteria, Lactobacillus, Bifidobacterium, and Desulfovibrio were reduced (Figure 6).

\section{Discussion}

Neurogastroenterology has revealed that there is direct biochemical signal transmission between the gastrointestinal tract and the central nervous system which is called the gutbrain axis [11]. Some reports have shown that gut microbiota can influence and regulate emotional behavior, which indicates that probiotic intake may provide effective ingredients to alleviate depressive symptoms [12]. The effect of probiotics on mental symptoms is related to the gut-brain axis, and the gut-brain axis can reduce systemic inflammation and regulate neurotransmission [13]. Although some animal models mimic depression phenotypes by inducing neuron changes similar to humans, the development of new treatments and biological mechanisms for depression may not be applicable to humans. However, under the premise 


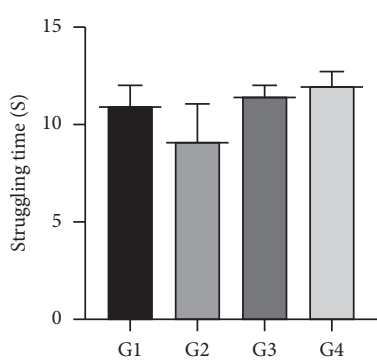

(a)

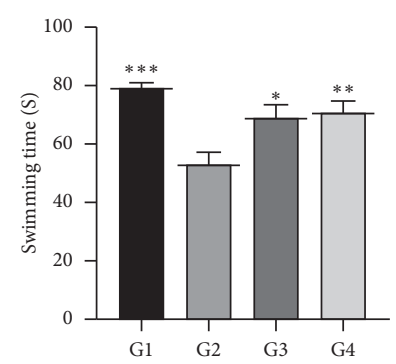

(b)

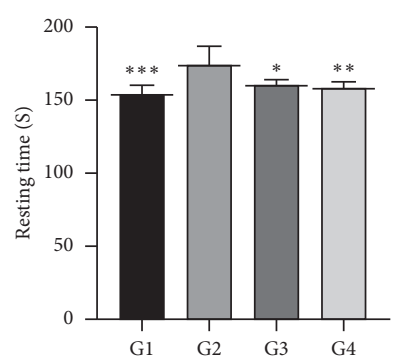

(c)

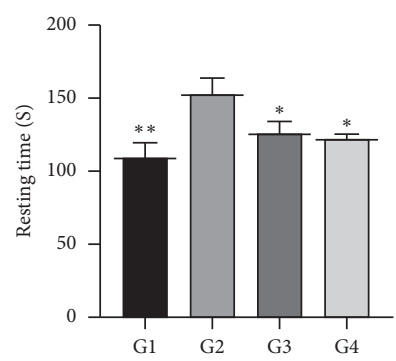

(d)

FIgURE 1: The behavioral comparison of rats in the forced swimming experiment and tail suspension experiment among four groups. (a) Struggling time of rats in FST. (b) Swimming time of rats in FST. (c) Resting time of rats in FST. (d) Resting time of rats in TST. ${ }^{*} 0.01<P<0.05,{ }^{* *} 0.01<P<0.001$, and ${ }^{* * *} P<0.001$.

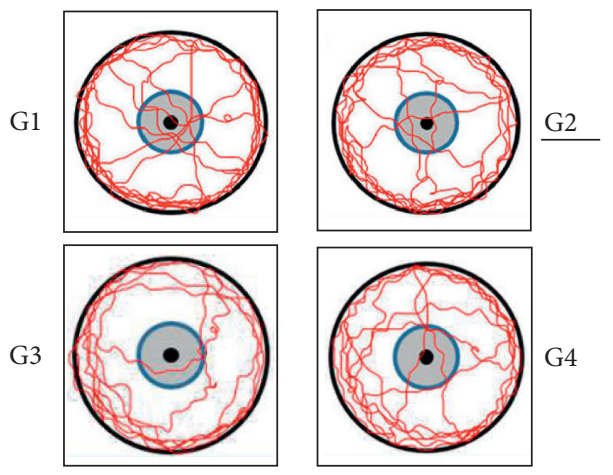

(a)

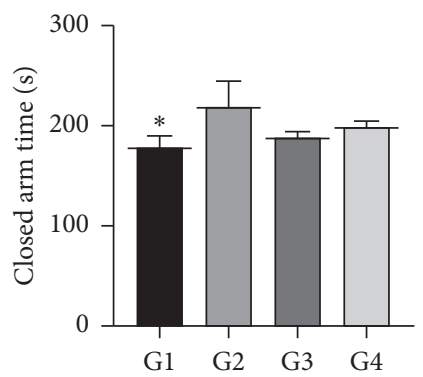

(d)
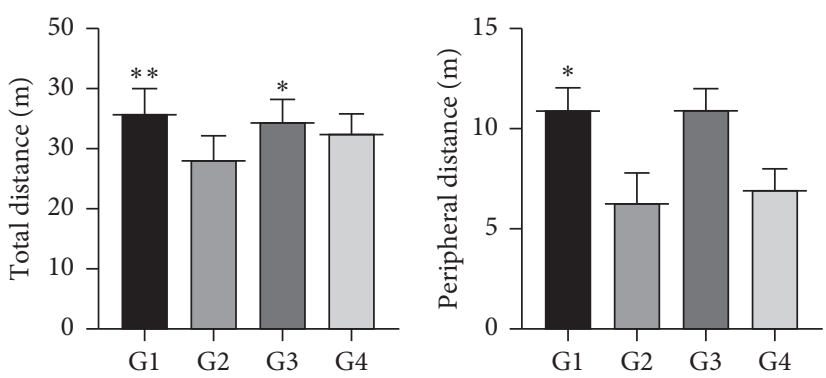

(c)

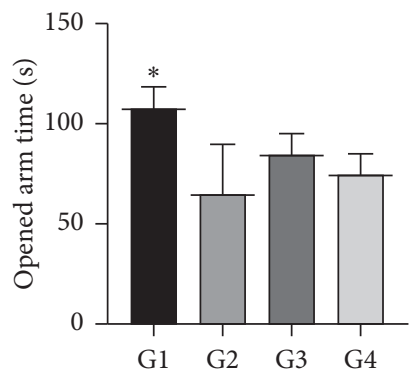

(e)

FiguRE 2: The behavioral comparison of rats in the open field experiment and elevated maze experiment among four groups. (a) The trajectory of rats in OFT. (b) The total distance of rats walk in the container in OFT. (c) The peripheral distance of rats walk in the container in OFT. (d) The time of rats spent in the closed arm of EPM. (e) The time of rats spent in the opened arm of EPM. (f) The difference of corticosterone concentration in serum of four groups of rats. ${ }^{*} 0.01<P<0.05$ and ${ }^{* *} 0.01<P<0.001$.

that animal models can provide treatment strategies that are suitable for humans, the molecular and cellular mechanisms for detecting depressive symptoms in a controlled environment are reliable.

Some reports have revealed the effect of regular physical exercise on the relief of mental symptoms $[14,15]$. In a rat model, aerobic exercise can reverse depression by regulating the hypothalamus-pituitary-adrenal axis [16-18]. In another rat model, regular physical exercise may increase the level of tryptophan in plasma and decrease the level of 5-hydroxytryptamine in the frontal cortex, thus alleviating the symptoms of depression [19, 20]. In addition, regular physical exercise of medium-low load can directly change the physical condition by increasing the proportion of rhamnose JB- 1 and enhancing its effect on $\gamma$-aminobutyric acid receptor expression to reduce depression-related behavior [21]. According to our observations, in the rat model, the same regular physical exercise can reduce depressionlike behavior, such as regular swimming. A recent report also showed that anxiety and depression-like behaviors induced by chronic mild stress can be improved by performing three regular physical exercises: swimming, treadmill running, 


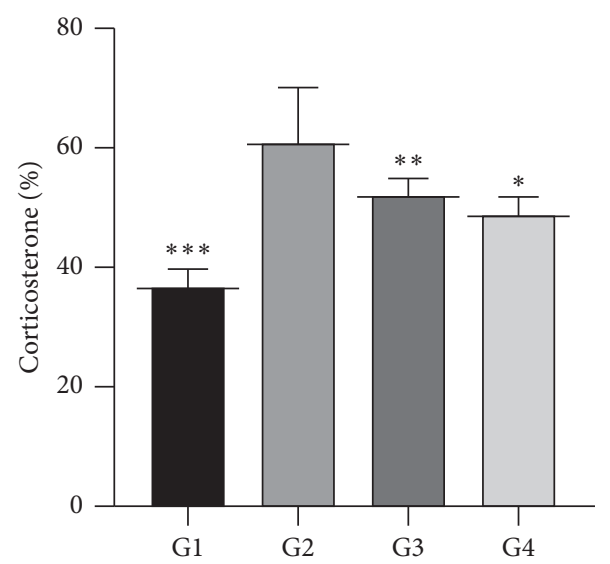

Figure 3: The difference of corticosterone concentration in serum of four groups of rats. ${ }^{*} 0.01<P<0.05,{ }^{* *} 0.01<P<0.001$, and ${ }^{* * *} P<0.001$.

TABLE 1: Alpha diversity of fecal microbiota in four groups of rats.

\begin{tabular}{lcccc}
\hline \multirow{2}{*}{ Group } & \multicolumn{2}{c}{ Richness estimates } & \multicolumn{2}{c}{ Diversity estimates } \\
G1 & Observed_species & Chao 1 & Shannon & Simpson \\
G2 & 7732.5 & 39788.96 & 7.042 & 0.972 \\
G3 & 8809.0 & 41881.26 & 7.586 & 0.980 \\
G4 & 8232.5 & 39164.41 & 7.216 & 0.970 \\
\hline
\end{tabular}

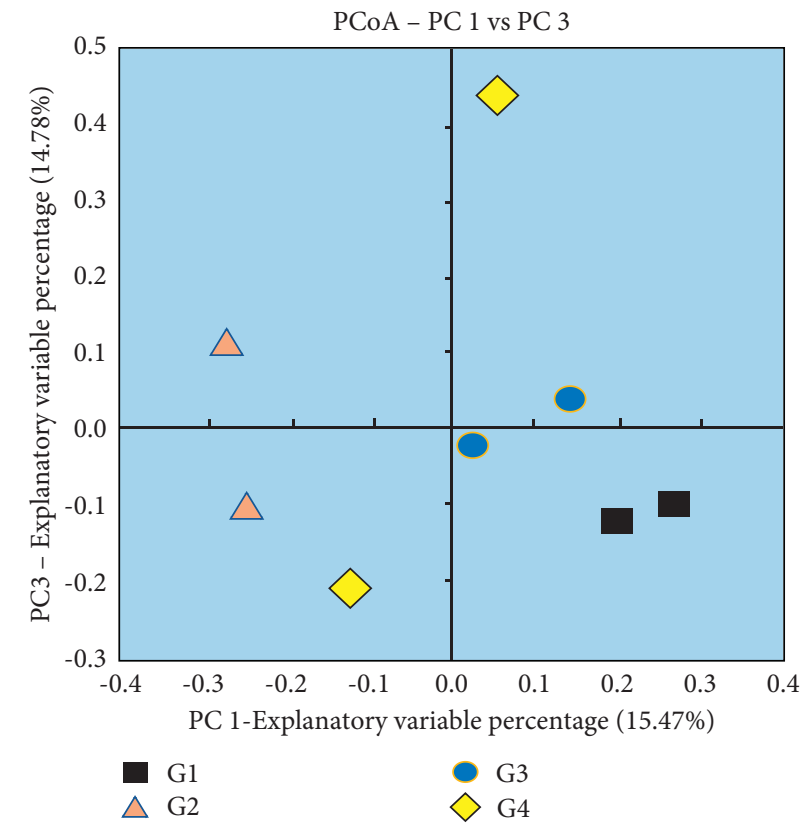

FIGURE 4: Principal component analysis (PCA) of fecal microbiota in four groups of rats. There are 10 rats in each group. G1, normal group; G2, chronic unpredictable mild stress used to develop depression-like behavior in rats; G3, fluoxetine was given $3 \mathrm{mg} / \mathrm{kg}$ daily by gavage after the formation of depression-like behavior; G4, the depressed model rats exercised for 1 hour at 9 am and 3 pm each day.

and weight-bearing ladder climbing [22, 23]. In addition, regular physical exercise can also relieve depression and anxiety. In the regular jogging and swimming treatment group, psychological pain was significantly reduced [24]. At the same time, the anxiety symptoms of patients with chronic fatigue syndrome undergoing aerobic physical exercise were significantly lower than those of the control group. These positive findings indicate that the strategy of 


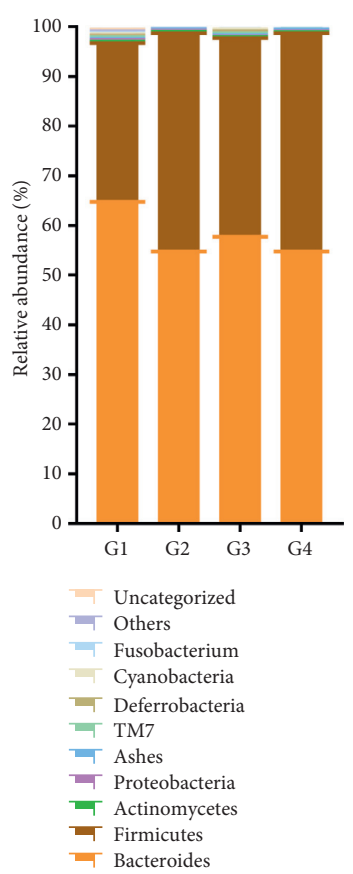

(a)
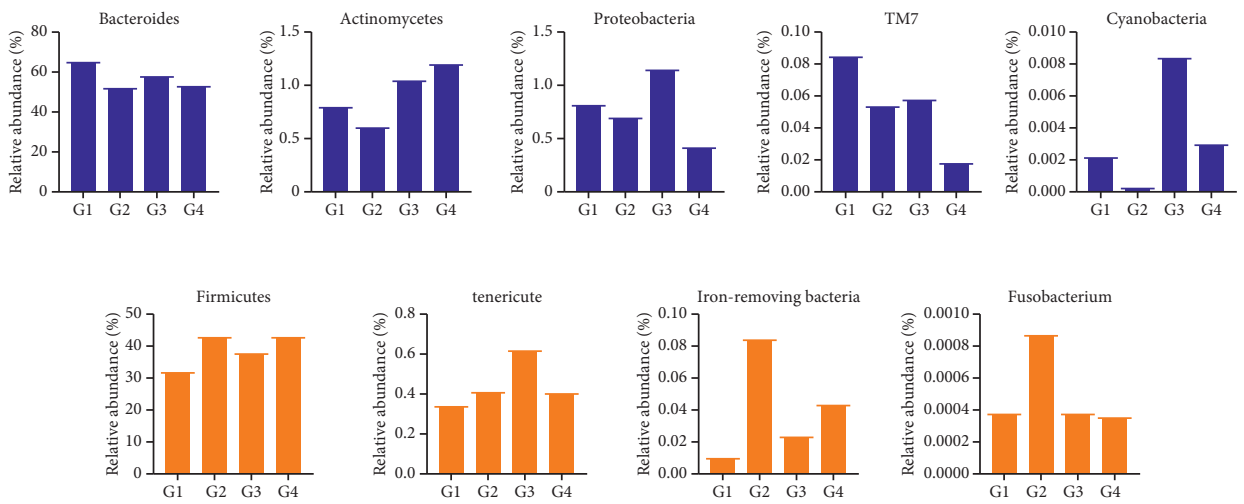

(b)

Figure 5: Phylum horizontal abundance of gut microbiota. (a) The different composition of the gut microbiota in four groups of rats. (b) Changes in various types of gut microbiota in four groups of rats.
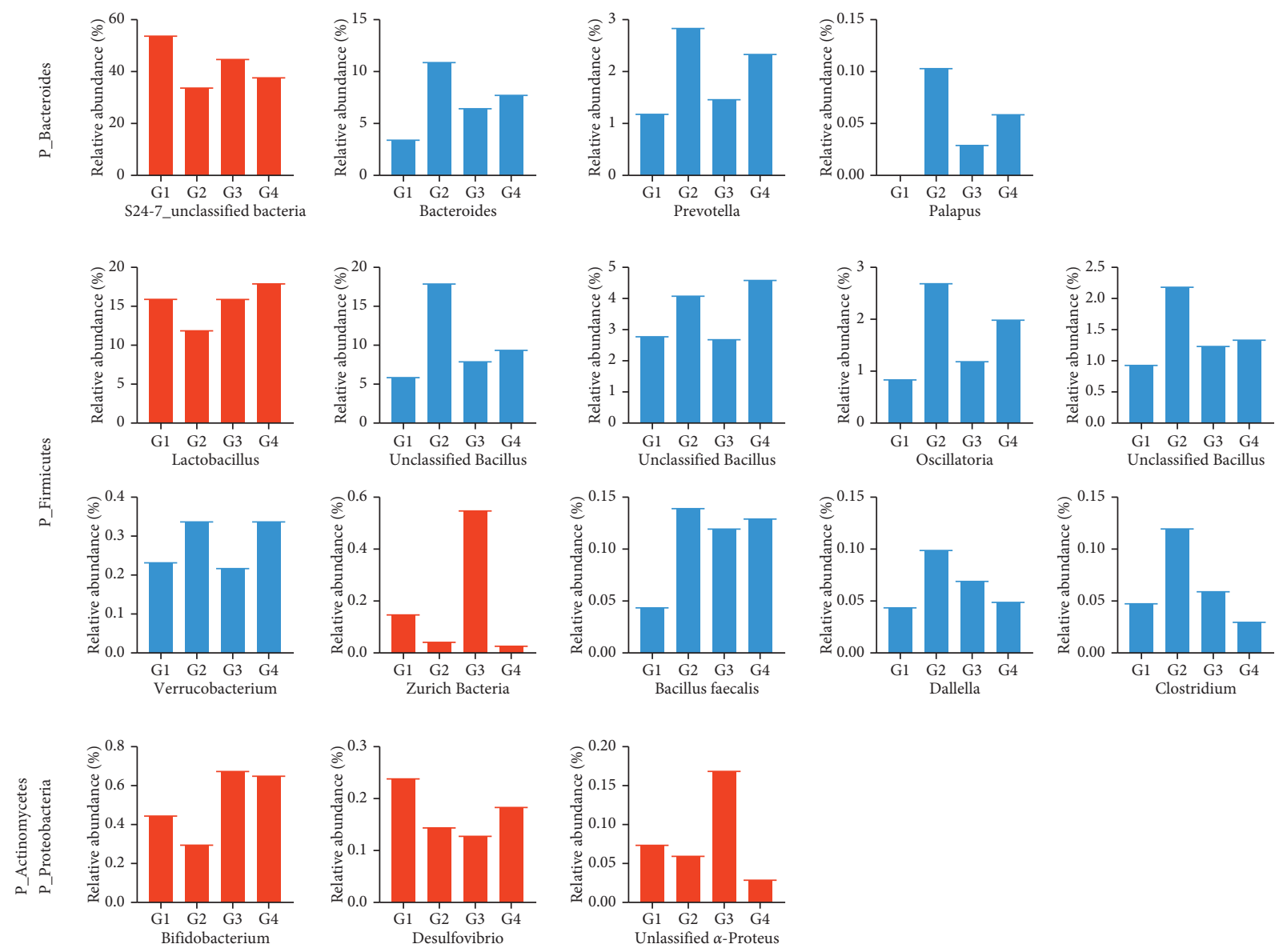

Figure 6: Genus horizontal abundance of gut microbiota. 
physical exercise therapy has great prospects [25]. In such strategies, regular physical exercise may become a target for the treatment of depression [26].

Although the role of regular physical exercise in improving the type and function of gut microbiota to improve depressive disorder has not been precisely explored, our research shows that the characteristics of gut microbiota imbalance are significantly different in taxonomy between G1 and G2. At the phylum level, the proportion of Actinomycetes in G2 decreased significantly, while the proportion of Actinomycetes in G4 increased significantly. The reason for this difference may be that regular physical exercise can improve the efficiency of the delivery of Bifidobacteria-containing probiotics to the gut, which in turn will increase the abundance of Actinomycetes in G4 [27]. It has been reported that there are significant differences in the proportions of Actinomycetes and Firmicutes in the composition of the gut microbiota between patients with severe depression and healthy people [28-30]. However, in this study, the proportion of Firmicutes did not recover after regular physical exercise. In addition, gut inflammation may be involved in the pathogenesis of depression [31]. Although the results obtained from this study do not show that regular physical exercise can alleviate gut inflammation in rats, we speculate that regular physical exercise can promote the anti-inflammatory activity of probiotics in the gut, protect gut barrier function, regulate inflammatory mediators, and thus reduce the symptoms of depression [32].

In conclusion, regular physical exercise can regulate stress-related behavior in rats. Because regular physical exercise changes the distribution of gut microbiota, this study may support that interventions based on intestinal microbiota can alleviate stress-related depression symptoms. Further extensive studies combining metagenomics and metabonomics are needed to explain the relationship between depression and regular physical exercise. However, based on our observations, we can clarify the partial relationship between the types and functional changes of intestinal microbiota induced by regular physical exercise and depression.

\section{Conflicts of Interest}

The authors declare that they have no conflicts of interest.

\section{Authors' Contributions}

Liangju Sheng and Yuxuan Wang contributed equally to this work, and they designed and ran the experiment. Yuxuan Wang, Yan Zhou, and Anqi Jiang wrote and reviewed the manuscript. Hong Zhou provided technical support.

\section{Acknowledgments}

This work was funded by the National Natural Science Foundation of China (81301194). The authors are grateful for the support of the fund.

\section{References}

[1] C. Ménard, G. E. Hodes, and S. J. Russo, "Pathogenesis of depression: insights from human and rodent studies," $\mathrm{Neu}$ roscience, vol. 321, pp. 138-162, 2016.

[2] E. A. Mayer, K. Tillisch, and A. Gupta, "Gut/brain axis and the microbiota," Journal of Clinical Investigation, vol. 125, no. 3 , pp. 926-938, 2015.

[3] F. Boem and A. Amedei, "Healthy axis: towards an integrated view of the gut-brain health," World Journal of Gastroenterology, vol. 25, no. 29, pp. 3838-3841, 2019.

[4] J. López-Torres Hidalgo, "Effectiveness of physical exercise in the treatment of depression in older adults as an alternative to antidepressant drugs in primary care," BMC Psychiatry, vol. 19, no. 1, p. 21, 2019.

[5] P. Zheng, B. Zeng, C. Zhou et al., "Gut microbiome remodeling induces depressive-like behaviors through a pathway mediated by the host's metabolism," Molecular Psychiatry, vol. 21, no. 6, pp. 786-796, 2016.

[6] J. R. Kelly, Y. Borre, C. O’ Brien et al., "Transferring the blues: depression-associated gut microbiota induces neurobehavioural changes in the rat," Journal of Psychiatric Research, vol. 82, pp. 109-118, 2016.

[7] G. Chevalier, E. Siopi, L. Guenin-Macé et al., "Effect of gut microbiota on depressive-like behaviors in mice is mediated by the endocannabinoid system," Nature Communications, vol. 11 , no. 1 , p. $6363,2020$.

[8] F. B. Schuch, D. Vancampfort, J. Richards, S. Rosenbaum, P. B. Ward, and B. Stubbs, "Exercise as a treatment for depression: a meta-analysis adjusting for publication bias," Journal of Psychiatric Research, vol. 77, pp. 42-51, 2016.

[9] S. Gujral, H. Aizenstein, C. F. Reynolds, M. A. Butters, and K. I. Erickson, "Exercise effects on depression: possible neural mechanisms," General Hospital Psychiatry, vol. 49, pp. 2-10, 2017.

[10] A. Sierakowiak, A. Mattsson, M. Gómez-Galán et al., "Hippocampal morphology in a rat model of depression: the effects of physical activity," The Open Neuroimaging Journal, vol. 9, no. 1, pp. 1-6, 2015.

[11] P. Holzer and A. Farzi, "Neuropeptides and the microbiotagut-brain Axis," Advances in Experimental Medicine \& Biology, vol. 817, pp. 195-219, 2014.

[12] S. Mörkl, M. I. Butler, A. Holl, J. F. Cryan, and T. G. Dinan, "Probiotics and the microbiota-gut-brain Axis: focus on psychiatry," Current Nutrition Reports, vol. 9, no. 3, pp. 171-182, 2020.

[13] J. Sanchez-Ramos, S. Song, V. Sava et al., "Granulocyte colony stimulating factor decreases brain amyloid burden and reverses cognitive impairment in Alzheimer's mice," Neuroscience, vol. 163, no. 1, pp. 55-72, 2009.

[14] A. L. Ridgel, B. L. Walter, C. Tatsuoka et al., "Enhanced Exercise Therapy in Parkinson's disease: a comparative effectiveness trial," Journal of Science and Medicine in Sport, vol. 19, no. 1, pp. 12-17, 2016.

[15] K. M. Tye, J. J. Mirzabekov, M. R. Warden et al., "Dopamine neurons modulate neural encoding and expression of depression-related behaviour," Nature, vol. 493, no. 7433, pp. 537-541, 2013.

[16] N. Mcneal, K. M. Appleton, A. K. Johnson et al., "The protective effects of social bonding on behavioral and pituitaryadrenal axis reactivity to chronic mild stress in prairie voles," Stress: The International Journal on the Biology of Stress, vol. 20, no. 2, pp. 175-182, 2017. 
[17] M. Wang, W. Dong, R. Wang et al., "Gastrodiae rhizoma water extract ameliorates hypothalamic-pituitary-adrenal Axis hyperactivity and inflammation induced by chronic unpredictable mild stress in rats," BioMed Research International, vol. 2020, Article ID 8374614, 7 pages, 2020.

[18] C. Zhang, Y.-P. Zhang, Y.-Y. Li et al., "Minocycline ameliorates depressive behaviors and neuro-immune dysfunction induced by chronic unpredictable mild stress in the rat," Behavioural Brain Research, vol. 356, pp. 348-357, 2019.

[19] H. Lee, M. Ohno, S. Ohta, and T. Mikami, "Regular moderate or intense exercise prevents depression-like behavior without change of hippocampal tryptophan content in chronically tryptophan-deficient and stressed mice," PLoS One, vol. 8, no. 7, Article ID e66996, 2013.

[20] L. Micheli, M. Ceccarelli, G. D'Andrea, and F. Tirone, "Depression and adult neurogenesis: positive effects of the antidepressant fluoxetine and of physical exercise," Brain Research Bulletin, vol. 143, pp. 181-193, 2018.

[21] P. J. Mueller and N. A. Mischel, "Selective enhancement of glutamate-mediated pressor responses after GABAA receptor blockade in the RVLM of sedentary versus spontaneous wheel running rats," Frontiers in Physiology, vol. 3, p. 447, 2012.

[22] S. Lapmanee, J. Charoenphandhu, J. Teerapornpuntakit, N. Krishnamra, and N. Charoenphandhu, "Agomelatine, venlafaxine, and running exercise effectively prevent anxietyand depression-like behaviors and memory impairment in restraint stressed rats," PLoS One, vol. 12, no. 11, Article ID e0187671, 2017.

[23] C. Yang, Z. Zhang, J. Ma et al., "Effects of different spectral energy distributions on physiological behavior and hormone levels in depression[J]," Iranian Journal of Public Health, vol. 47 , no. 3, pp. 373-381, 2018.

[24] M. Toups, T. Carmody, T. Greer, C. Rethorst, B. Grannemann, and M. H. Trivedi, "Exercise is an effective treatment for positive valence symptoms in major depression," Journal of Affective Disorders, vol. 209, pp. 188-194, 2017.

[25] J. Krogh, C. Hjorthøj, H. Speyer, C. Gluud, and M. Nordentoft, "Exercise for patients with major depression: a systematic review with meta-analysis and trial sequential analysis," BMJ Open, vol. 7, no. 9, Article ID e014820, 2017.

[26] M. Tang, H. Huang, S. Li et al., "Hippocampal proteomic changes of susceptibility and resilience to depression or anxiety in a rat model of chronic mild stress," Translational Psychiatry, vol. 9, no. 1, p. 260, 2019.

[27] H. Jiang, Z. Ling, Y. Zhang et al., "Altered fecal microbiota composition in patients with major depressive disorder," Brain, Behavior, and Immunity, vol. 48, pp. 186-194, 2015.

[28] L. Jianguo, J. Xueyang, W. Cui, W. Changxin, and Q. Xuemei, "Correction: altered gut metabolome contributes to depression-like behaviors in rats exposed to chronic unpredictable mild stress," Translational Psychiatry, vol. 9, no. 1, p. 275, 2019.

[29] B. Li, K. Guo, L. Zeng et al., "Metabolite identification in fecal microbiota transplantation mouse livers and combined proteomics with chronic unpredictive mild stress mouse livers," Translational Psychiatry, vol. 8, no. 1, p. 34, 2018.

[30] D. Kuti, Z. Winkler, K. Horváth et al., "Gastrointestinal (nonsystemic) antibiotic rifaximin differentially affects chronic stress-induced changes in colon microbiome and gut permeability without effect on behavior," Brain, Behavior, and Immunity, vol. 84, pp. 218-228, 2020.
[31] M. Grochowska, M. Wojnar, and M. Radkowski, "The gut microbiota in neuropsychiatric disorders," Acta Neurobiologiae Experimentalis, vol. 78, no. 2, pp. 69-81, 2018.

[32] A. M. Petersen and B. K. Pedersen, "The anti-inflammatory effect of exercise," Journal of Applied Physiology, vol. 98, no. 4, pp. 1154-1162, 1985. 